\title{
DUE CANTATE SPURIE DI FRANCESCO ANTONIO PISTOCCHI A MILANO
}

\author{
ALEJANDRA BÉJAR BARTOLO \\ Universidad de Guanajuato, México
}

\begin{abstract}
Izvleček: Analiza strukturnih značilnosti (zaporedja tonalitet, instrumentalnih uvodov in ariosov) in glasbenega jezika (posebnosti in napak $v$ harmoniji in kontrapunktu ter razmerja med besedilom in glasbo) dveh rokopisnih kantat za sopran, ohranjenih $v$ knjižnici milanskega konservatorija »Giuseppe Verdi«, kaže, da sta bili napačno pripisani Francescu Antoniu Pistocchiju.
\end{abstract}

Ključne besede: rokopis, avtorstvo, uglasbitev besedila, kontrapunkt, zaporedja tonalitet

\begin{abstract}
An analysis of the structural characteristics (key sequences, instrumental introductions, ariosi) and the musical language (peculiarities and errors in harmony and counterpoint and the text-music relationship) of two manuscript cantatas for soprano ascribed to Francesco Antonio Pistocchi that are preserved in the library of the Conservatorio di musica "Giuseppe Verdi" in Milan proves their incorrect attribution.

Keywords: manuscript, authorship, musical setting, counterpoint, key sequences
\end{abstract}

Il corpus delle cantate per voce sola e basso continuo del compositore Francesco Antonio Pistocchi (Palermo, 1659-Bologna, 1726) non è ancora stato indagato nel suo complesso, nonostante includa - allo stato attuale delle conoscenze - una quarantina di titoli. Un primo tentativo di ricognizione sistematica è stato realizzato soltanto pochi anni fa, ${ }^{1}$ mentre delle sole cantate pubblicate vivente l'autore (e giunte a noi) ${ }^{2}$ - cioè le prime sei composizioni degli Scherzi musicali, [op. 2] (Amsterdam: Estienne Roger, [1698]) esiste sia un'edizione critica moderna, ${ }^{3}$ sia uno studio analitico. ${ }^{4}$ Per contro, le cantate

1 Béjar Bartolo e Ammetto, Las composiciones de Francesco Antonio Pistocchi, 322-327.

2 È documentata l'esistenza di una raccolta (perduta) di "Cantate di Fran. ${ }^{\text {co }}$ Ant. ${ }^{\circ}$ Pistocchi op. 1" (Bologna: Marino Silvani, 1698), menzionata nel catalogo dell'editore musicale e compositore bolognese Giuseppe Antonio Silvani (cfr. Silvani, Indice dell'opere di musica, n. 159; e Mischiati, Indici, cataloghi e avvisi degli editori, n. XIX). Non è chiara la ragione per la quale Pistocchi autorizzò la stampa di queste cantate come "op. 1", avendo già pubblicato tre decenni prima, e sempre a Bologna, i suoi Capricci puerili, op. 1 (Giacomo Monti, 1667): una possibile spiegazione potrebbe essere che l'op. 1 per Silvani non rappresentava la prima pubblicazione di Pistocchi, ma la sua 'prima' opera a stampa di cantate.

3 Pistocchi, Scherzi musicali, 15-26 (testi e apparati critici), 33-84 (partiture).

4 Béjar Bartolo e Ammetto, "Aspectos de análisis textual y musical", 8-13. Inoltre, alcuni singoli movimenti di questa collezione sono stati analizzati da Michael Talbot per aspetti particolari, in Talbot, Chamber Cantatas of Antonio Vivaldi, 53-53; e in Talbot, Aspects of the Secular Cantata, 262-266. 
manoscritte di Pistocchi sono state praticamente trascurate tanto dai musicologi quanto dagli interpreti, con pochissime eccezioni. ${ }^{5}$

La difficoltà per poter gettare uno sguardo complessivo su questa porzione significativa della produzione musicale di Pistocchi - storicamente importante in quanto registra significativi cambiamenti formali 'interni' al genere cantata, avvenuti nei decenni a cavallo del 1700 - è dovuta principalmente a due aspetti: da un lato, il problema del censimento e reperimento delle fonti, disseminate in oltre una ventina di biblioteche di vari paesi d'Europa (Austria, Francia, Germania, Gran Bretagna, Italia, Spagna) ${ }^{6}$ e, dall'altro, la saltuaria presenza di attribuzioni autoriali discordanti nelle fonti che trasmettono una stessa composizione. ${ }^{7}$ Riguardo a quest'ultimo aspetto, per esempio, una decina di cantate risultano attribuite tanto a Pistocchi come ad altri compositori: è il caso di Amorosa pastorella (Alessandro Scarlatti), Come Dorinda, come (Severo de Luca), Ecco il sole, ecco il sol (Giovanni Bononcini), Gelsomino ridente (Giuseppe Porsile), Pallidetta viola (Carlo Francesco Cesarini), Per consolar mie pene (G. Bononcini), Per te forse, mio ben ([?] Mancini), Per un volto di gigli e di rose (Tomaso Albinoni e A. Scarlatti), Sovra un bel poggio assisa (G. Bononcini) e Tortorelle imprigionate (Andrea Stefano "Fiori" [Fiorè]). Se per alcune di queste cantate le differenti attribuzioni sono state risolte a favore di Pistocchi (Pallidetta viola, ${ }^{8}$ Per te forse, mio ben, ${ }^{9}$ Per un volto di gigli e di rose),${ }^{10} \mathrm{o}$ di

5 I primi due movimenti della cantata per contralto Oh, sempre al tuo pastore (Museo internazionale e biblioteca della musica di Bologna [I-Bc], DD.48) vennero pubblicati nel 1894 in un arrangiamento per canto e piano (Pistocchi, Frammento di una Cantata). Recentemente, la cantata Pallidetta viola - tramandata (sia per soprano, sia per contralto) da cinque fonti conservate in Francia, Gran Bretagna e Italia - è stata oggetto di uno studio che ha identificato la voce e la tonalità originali della composizione (Béjar Bartolo, "Una cantata, dos voces"). A livello discografico, invece, l'unica registrazione disponibile è ancora quella del 2001 (Pistocchi, Kantaten und Duette) che include due cantate manoscritte per soprano: Con dolce mormorio (I-Bc, DD.44, nel disco interpretata da una voce di contralto con trasposizione di tonalità, dall'originale la maggiore a mi bemolle maggiore) e Il giglio e la viola (Münster, Santini-Bibliothek [D-MÜs], SANT Hs 3222).

${ }^{6}$ Inoltre, ad oggi, più di una ventina di fonti di cantate di Pistocchi non sono state ancora censite dal RISM, e non è da escludere che ulteriori manoscritti possano in futuro venire alla luce.

7 Alcune cantate sono oggi note attraverso quattro, cinque o, in un caso, addirittura otto fonti distinte (Per un volto di gigli e di rose).

8 L'errata attribuzione a C. F. Cesarini, attestata soltanto in una delle cinque fonti che trasmettono questa composizione, è dovuta probabilmente a Francesco [Antonio] Lanciani, copista del volume miscellaneo di cantate - conservato a Genova - che ospita Pallidetta viola. La composizione è disponibile in un'edizione critica che descrive e analizza tutte le fonti note (Pistocchi, Chamber Cantata "Pallidetta viola").

9 Due fonti attribuiscono la composizione a Pistocchi (The British Library [GB-Lbl], Add. 62102; e Padova, Pontificia Biblioteca Antoniana [I-Pca], D.I.1367), mentre in un terzo manoscritto (Sondershausen, Stadtbibliothek "Johann Karl Wezel” [D-SHs], Mus B1:1) l'autore indicato è un non meglio precisato "Sig. ${ }^{\mathrm{r}}$ Mancini".

${ }^{10}$ Cinque fonti attribuiscono la composizione a Pistocchi (Österreichische Nationalbibliothek, Musiksammlung [A-Wn], E.M. 178; GB-Lbl, Add. 31503; GB-Lbl, Add. 34053; London, Gresham College [GB-Lgc], G. Mus. 362 (vol. 1); I-Bc, V.292), mentre due la assegnano erroneamente ad Albinoni (Staatsbibliothek zu Berlin - Preußischer Kulturbesitz, Musikabteilung [D-B], Mus.ms. 
altri compositori (Sovra un bel poggio assisa), ${ }_{11}^{11}$ per le rimanenti composizioni persistono tuttora alcune incertezze.

Infine, un ulteriore aspetto problematico per la definizione della paternità di una cantata, qualora venga attribuita a un solo autore in una o in più fonti, è quello relativo a particolari caratteristiche interne alla composizione che ne potrebbero mettere in discussione l'autenticità: in questo studio verranno prese in considerazione due cantate manoscritte appartenenti a questa tipologia.

\section{Le fonti manoscritte}

Due cantate per soprano e basso continuo, ascritte a Pistocchi, sono conservate nel fondo Noseda della Biblioteca del Conservatorio di musica "Giuseppe Verdi" di Milano: ${ }^{12}$ si tratta di Come Dorinda, come e E mi lasciasti, ingrata. Della prima composizione esiste anche una concordanza che la attribuisce invece al compositore napoletano Severo de Luca ( $f$. 1684-1734), autore di varie cantate per soprano. Ecco una descrizione sintetica delle fonti:

\section{Come Dorinda, come}

- Milano, Biblioteca del Conservatorio di musica "Giuseppe Verdi" (I-Mc), Noseda O.31.13, cc. 1r-3v (la c. 1r è vuota), ${ }^{13}$ RISM: deest. Partitura di formato oblungo $(21,4 \times$ $27,3 \mathrm{~cm}$ ) con dieci pentagrammi per pagina. Titolo: "Cantata à Voce Sola. Del Sig. Pistochini" [sic], in sol minore. Struttura: ${ }^{14} \operatorname{Rec}$ [itati]vo, Aria, Rec[itati]vo, Aria.${ }^{15}$

- Napoli, Biblioteca del Conservatorio di musica S. Pietro a Majella (I-Nc), Cantate 105 (olim 34.4.14), cc. 1r-4v (la c. 4r-v è vuota), RISM: deest. ${ }^{16}$ Partitura di formato oblungo

30136 (5); Bibliothèque nationale de France, Département de la Musique [F-Pn], Rés Vmc Ms 81-6) e un'ulteriore fonte ad A. Scarlatti (London, Royal Academy of Music, Library [GB-Lam], MS 37). L'attribuzione a Scarlatti è stata messa in dubbio sia da Hanley, "Alessandro Scarlatti's cantate da camera", 548; sia da Rostirolla, Alessandro Scarlatti, 462, 500.

${ }^{11}$ Delle otto fonti che trasmettono questa cantata, sette la attribuiscono a G. Bononcini (GB-Lam, MS 34; GB-Lbl, Add. 62102; GB-Lbl, R.M.24.c.17; Oxford, Bodleian Library [GB-Ob], MS. Mus. d.20; GB-Ob, MS. Mus. d.22; Reggio nell'Emilia, Biblioteca Panizzi [I-Rem], Mus. Prof. 31; Chicago, The Newberry Library [US-Cn], VM1532.B69L), mentre soltanto una la considera erroneamente di Pistocchi (D-B, Mus.ms. 30197).

${ }^{12}$ Ringrazio la biblioteca del conservatorio milanese per avermi inviato una riproduzione delle due cantate.

${ }^{13}$ A c. $3 \mathrm{v}$, in basso a destra, si legge una data apposta con pennino: "1707".

${ }^{14}$ Si trascrive in corsivo la dicitura che compare nella fonte, e si scioglie l'abbreviatura tra parentesi quadre.

${ }^{15}$ Soltanto nel recitativo iniziale compaiono un paio di indicazioni relative al cifrato per il basso continuo.

${ }^{16}$ OPAC SBN, il "Catalogo del Servizio Bibliotecario Nazionale" italiano (opac.sbn.it), colloca la redazione del manoscritto a Napoli, nel primo decennio del Settecento. Una riproduzione della fonte è disponibile online: www.internetculturale.it. 
$(21 \times 26 \mathrm{~cm})$ con dieci pentagrammi per pagina. Titolo: "Cantata del Sig. ${ }^{r}$ Severo de Luca", in mi minore. Struttura: [Recitativo], Aria, [Recitativo], [Aria]. ${ }^{17}$

\section{E mi lasciasti, ingrata}

- Milano, Biblioteca del Conservatorio di musica "Giuseppe Verdi" (I-Mc), Noseda O.31.14, cc. 1r-6v (la c. 6v è vuota), RISM: deest. ${ }^{18}$ Partitura di formato oblungo $(21,5 \times$ $28,3 \mathrm{~cm}$ ) con otto pentagrammi per pagina. Titolo: "Cantata à voce Sola | Del Sig. ${ }^{r}$ Pistocchino", in la maggiore. Struttura: [Recitativo], Aria, Rec[itativo], Aria. Andante. ${ }^{19}$

\section{I testi poetici}

Le cantate oggetto di questo studio sono entrambe articolate nella struttura RARA, ${ }^{20}$ sebbene a livello poetico mostrino alcune differenze, sia nelle lunghezze dei testi, sia nell'impiego di differenti tipi di versi per le arie: ottonari ${ }^{21}$ e senari ${ }^{22}$ in Come Dorinda, come, settenari ${ }^{23}$ e quinari ${ }^{24}$ in E mi lasciasti, ingrata. Nei recitativi, invece, vengono alternati - come di prassi - settenari ed endecasillabi (anche se in Come Dorinda, come il verso di undici sillabe metriche viene impiegato soltanto alla fine di ciascuno dei due recitativi).

Di seguito si trascrivono i testi delle due cantate, ${ }^{25}$ ai quali è stata aggiunta la punteggiatura, quasi completamente assente nelle fonti (della prima cantata, in particolare).

${ }^{17}$ Il manoscritto non registra indicazioni di cifrato per il basso continuo.

${ }^{18}$ OPAC SBN data il manoscritto nel periodo 1661-1690.

${ }^{19}$ In alcuni passaggi della parte del basso è attestato l'utilizzo della chiave di tenore (cfr. le batt. 11-12 della prima aria e le batt. 1-6 della seconda aria); inoltre, in tutti i movimenti sono presenti indicazioni relative al cifrato per la realizzazione del basso continuo.

${ }^{20}$ Si utilizzano le abbreviature R per 'recitativo', A per 'aria', seguite da un numero arabo (1 o 2) per specificare la posizione del movimento all'interno della composizione.

${ }^{21}$ Con rime axbx.

${ }^{22}$ Con rime aax, cddx.

${ }^{23}$ Con rime abx, abx.

${ }^{24}$ Con rime abcx, bacx.

${ }^{25}$ Ringrazio Fabrizio Ammetto e Giacomo Gibertoni per la revisione dei testi poetici. 
Come Dorinda, come

R1 Come Dorinda, come temi che lontananza possa del tuo bel nome farmi scordare e della tua sembianza?

A1 Ah, t'inganni o bella mia, ah, che pria morir saprò ch'io mi scordi ${ }^{26}$ de' tuoi rai, no, che mai non lo farò!

R2 Porto meco li strali

delle tue luci vaghe, onde sanar le piaghe già mai non si potranno se i dardi de' tuoi sguardi sempre in mezzo del cor fissi mi stanno.

A2 In ogni momento

di te mi rammento, amato mio ben.

Ti brama, ti chiama, ti cerca il mio core, che acceso $^{27}$ d'amore mi palpita in sen.

\section{E mi lasciasti, ingrata}

R1 E mi lasciasti, ingrata, e meco pur lasciasti la rimembranza, oh Dio, del tuo bel volto! E come il cor disciolto non mi lasciasti ancor? Come, spietata, sol mi lasciasti a tormentarmi il core?

La memoria infelice del tuo bel seno e del mio fido amore come soffrir mi lice d'amor l'ardente face senza te, senza core e senza pace!

26 I-Mc: "scorda".

27 I-Mc: "ch'acceso". 
A1 Lo sai, crudel, ch'io t'amo

e fiera pur mi fuggi

e vai lontan da me.

Mia vita, se ti chiamo,

perché, perché mi struggi,

barbara senza fé?

R2 Torna, deh, torna, oh Dio,

rendi lo spirto al sen, al cor la calma!

Quando per te la spero

ti siegua innamorata,

oh, mia crudele e fuggitiva amante,

rispondi al mio sospiro

con cui spesso ti chiama il cor costante!

$\mathrm{Ah}$, non far che vagante

sia come il tuo bel piede anche il pensiero!

Torna e del ciglio arciero

vedi come in trionfo arde d'amore

questo, tuo non più, mio povero core!

A2 Un'altra volta,

s'io ti rimiro,

il cor contento

si renderà.

$\mathrm{E}$ in un sospiro

l'alma disciolta

del suo tormento

goder saprà. 


\section{Gli incipit musicali}

A continuazione, si trascrivono gli incipit musicali (1-8) relativi alle intonazioni dei primi versi di ogni movimento delle due cantate: di seguito all'indicazione di ciascun movimento si indica il numero complessivo delle battute. Vengono rispettate le armature di chiave originali presenti nei manoscritti. Le alterazioni tra parentesi sono assenti nella fonte, o segnalano un accidente 'di cortesia'. Nel caso di Come Dorinda, come vengono annotate le versioni di entrambe le fonti (I-Mc e I-Nc).

\section{Incipit musicale 1}

Come Dorinda, come, recitativo I "Come Dorinda, come", 5 batt.

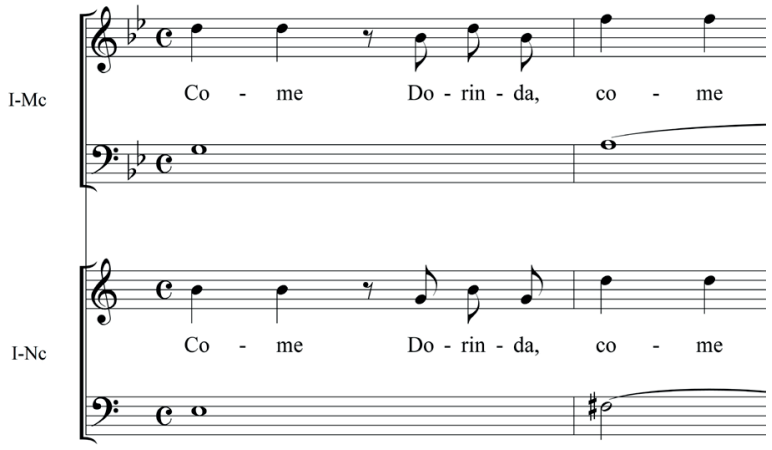

\section{Incipit musicale 2}

Come Dorinda, come, aria I "Ah, t'inganni o bella mia", ${ }^{28} 59$ batt. ${ }^{29}$ (indicazione "Da Capo" 30 dopo batt. 41)

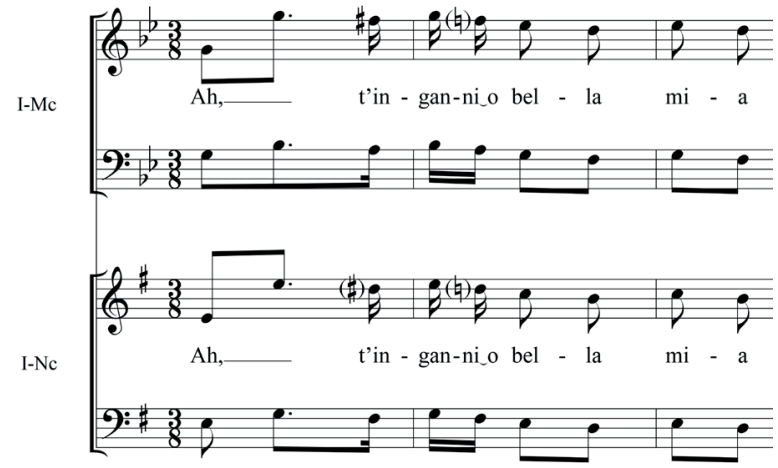

${ }^{28}$ In I-Nc, batt. 1, le prime due note del soprano sono senza travatura, mentre la prima nota del basso continuo è una semiminima (invece di una croma).

${ }^{29}$ Per il numero totale delle battute di questa aria nella fonte I-Mc si veda il paragrafo relativo agli aspetti musicali.

${ }^{30}$ In I-Nc si legge "da Capo sub. ${ }^{\circ}[=$ subito]". 


\section{Incipit musicale 3}

Come Dorinda, come, recitativo II "Porto meco li strali", 7 batt.

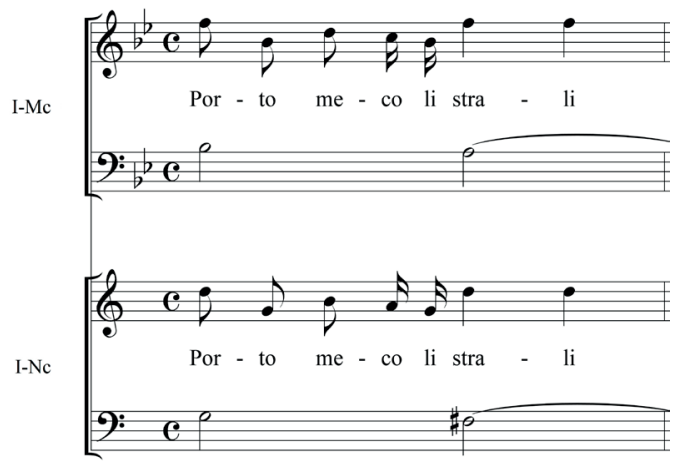

\section{Incipit musicale 4}

Come Dorinda, come, aria II "In ogni momento", 31 batt. (indicazione "Da Capo"31 dopo batt. 18)

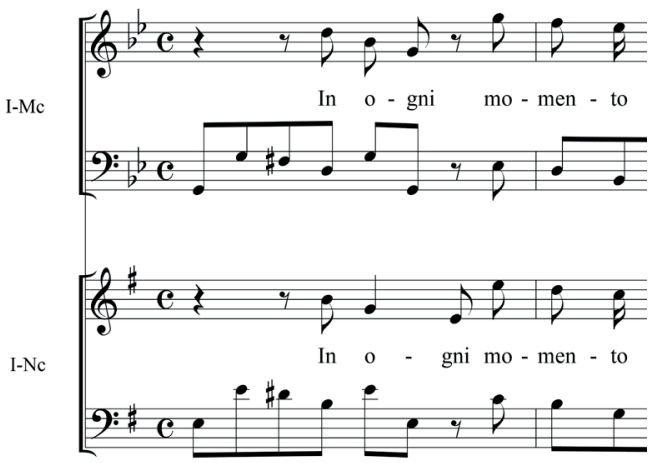

\section{Incipit musicale 5}

E mi lasciasti, ingrata, recitativo I "E mi lasciasti, ingrata", 16 batt.

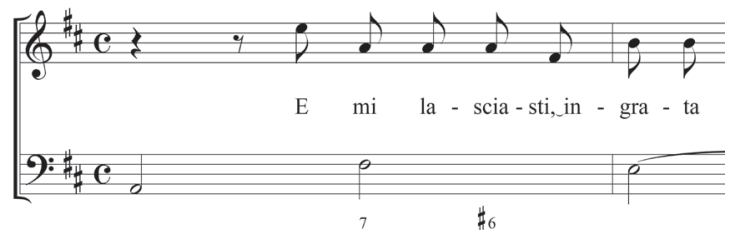

${ }^{31}$ In I-Nc si legge "da Capo sub.". 
Incipit musicale 6

E mi lasciasti, ingrata, aria I "Lo sai, crudel, ch'io t'amo", 35 batt. (indicazione "d[a] Capo" dopo batt. 22/I)

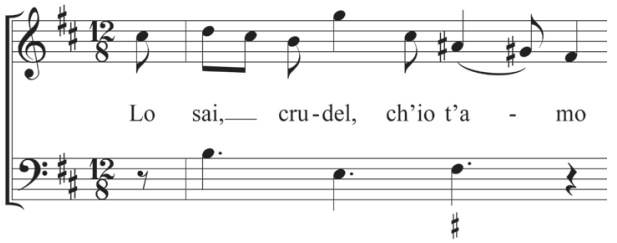

Incipit musicale 7

E mi lasciasti, ingrata, recitativo II "Torna, deh, torna, oh Dio", 17 batt.

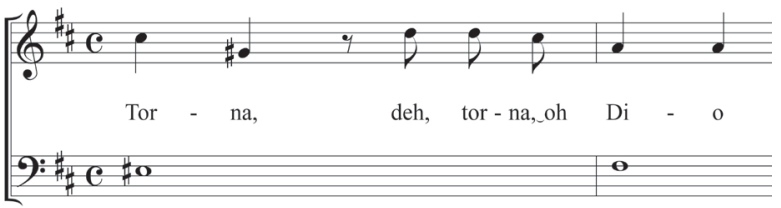

\section{Incipit musicale 8}

E mi lasciasti, ingrata, aria II "Un'altra volta”, 97 batt. (indicazione "d[a] Capo" dopo batt. 58)

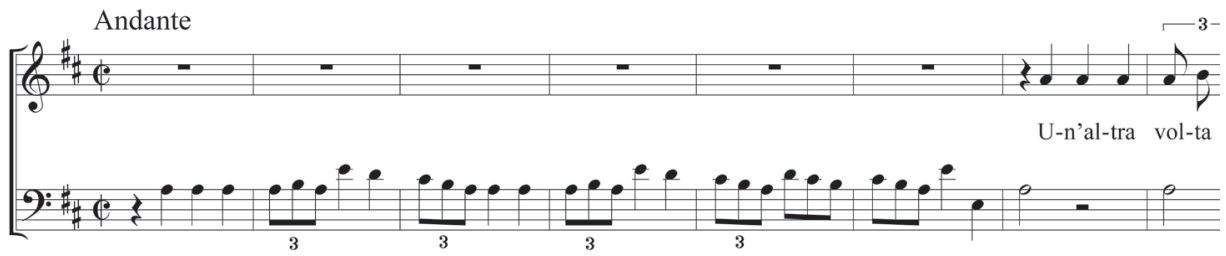

\section{Problemi testuali nelle fonti della cantata Come Dorinda, come}

Entrambe le fonti che trasmettono il testo della cantata Come Dorinda, come rivelano una scarsa accuratezza da parte dei due differenti copisti, per la presenza di alcuni errori di note (o di valori) e omissioni di alterazioni.

In I-Nc, oltre alle imprecisioni già evidenziate nell'incipit della prima aria, si segnala anche la mancanza del diesis davanti all'ultima nota di batt. 8 della linea vocale; nel secondo recitativo la nota finale del basso è erroneamente $\mathrm{Fa}_{1}$ (invece di Sol ); nell'aria conclusiva, sempre nella parte del basso, l'ultima nota di batt. 13 e la terza nota della misura seguente sono entrambe $\mathrm{Mi}_{2}$ (invece di $\mathrm{Re}_{2}$ ); infine, sempre nella stessa aria, batt. 17, la penultima nota della parte vocale è priva del diesis.

Anche I-Mc presenta alcuni errori o dimenticanze: nella seconda aria, la penultima nota di batt. 9 nella linea vocale è $\mathrm{Fa}_{3}$ (invece di Sol $\mathrm{S}_{3}$, mentre la prima nota di batt. 17 del basso è priva del bequadro; nella prima aria si registra addirittura la completa omissione di copiatura delle batt. 13 e 32-34, che ha provocato uno slittamento in avanti del testo verbale sottoposto alla musica, oltre ad altri errori armonico-contrappuntistici (a batt. 39). 


\section{Esempio musicale 1}

Come Dorinda, come, aria "Ah, t'inganni o bella mia", batt. 36-40

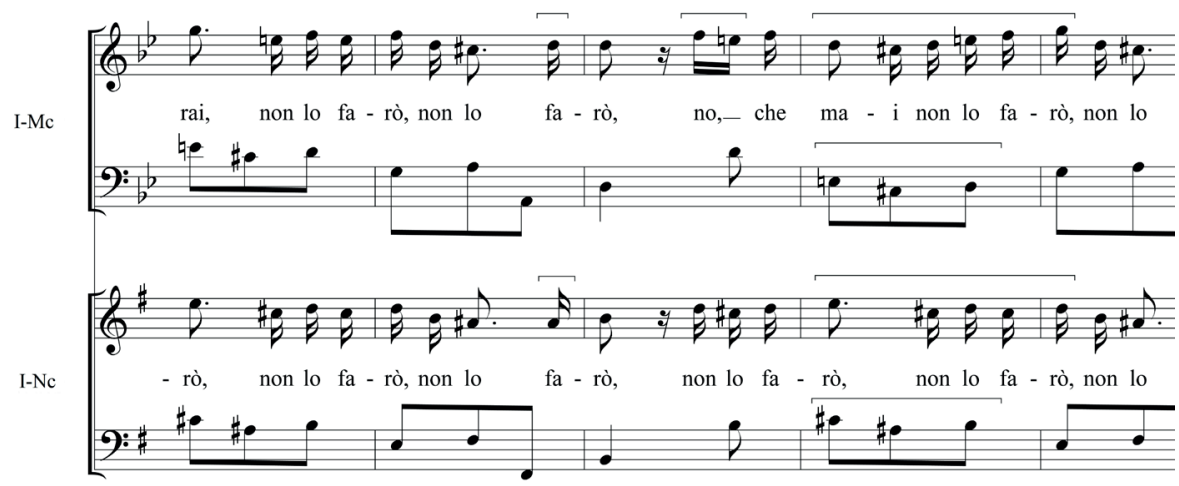

Un confronto tra le lezioni di alcuni passaggi tramandati dalle due fonti, relativi a cambi di ottava nella linea del basso, suggerisce che la versione in mi minore (I-Nc) possa essere una trasposizione - non sempre perfettamente riuscita - dalla tonalità (originale?) di sol minore (I-Mc): si vedano, in proposito, le batt. 3-5 (ove a batt. 3 compare un maldestro salto ascendente $\mathrm{Re}_{2}-\mathrm{Do}_{3}$ ) e 24 nella prima aria, o le batt. 1-2 e 11 nella seconda aria. ${ }^{32}$

\section{Esempio musicale 2}

Come Dorinda, come, aria "Ah, t'inganni o bella mia", batt. 3-5

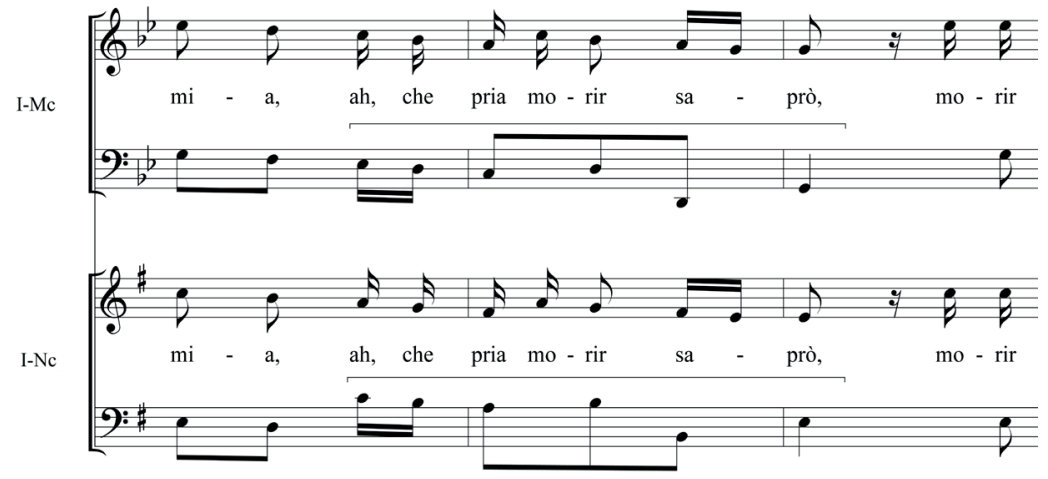

Altre piccole divergenze tra le due fonti, non classificabili come errori, sembrano invece attestare una trasmissione della composizione derivante da tradizioni differenti. A batt. 4 del secondo recitativo, le prime due crome della parte vocale in I-Mc sono attestate una terza sopra rispetto a I-Nc (entrambe le versioni sono armonicamente corrette). Nelle batt. 9 e 12 dell'aria conclusiva, la seconda nota delle prime tre crome nella linea

${ }^{32}$ Per contro, a batt. 39 di I-Mc, l'abbassamento di un'ottava delle tre crome del basso (cfr. Esempio musicale 1) sembra piuttosto dovuto a un ulteriore errore del copista, forse ancora confuso dall'omissione delle misure precedenti; d'altra parte, a batt. 36 compare la lezione musicale corretta dello stesso identico passaggio, tanto nella parte del canto (eccezione fatta per il testo sottoposto alla prima nota) come nella linea del basso. 
del basso presenta un salto di terza discendente in I-Mc $\left(\mathrm{Re}_{3}, \mathrm{Sib}_{2}, \mathrm{Re}_{3}\right)$, mentre è nota di volta in I-Nc $\left(\mathrm{Si}_{2}, \mathrm{La}_{2}, \mathrm{Si}_{2}\right)$ : pure in questo caso le due lezioni sono entrambe valide. Infine, anche le ultime due misure della sezione centrale della stessa aria registrano nella linea del basso due lezioni alternative (a batt. 17 e a batt. 18).

\section{Esempio musicale 3}

Come Dorinda, come, aria "In ogni momento", batt. 17-18

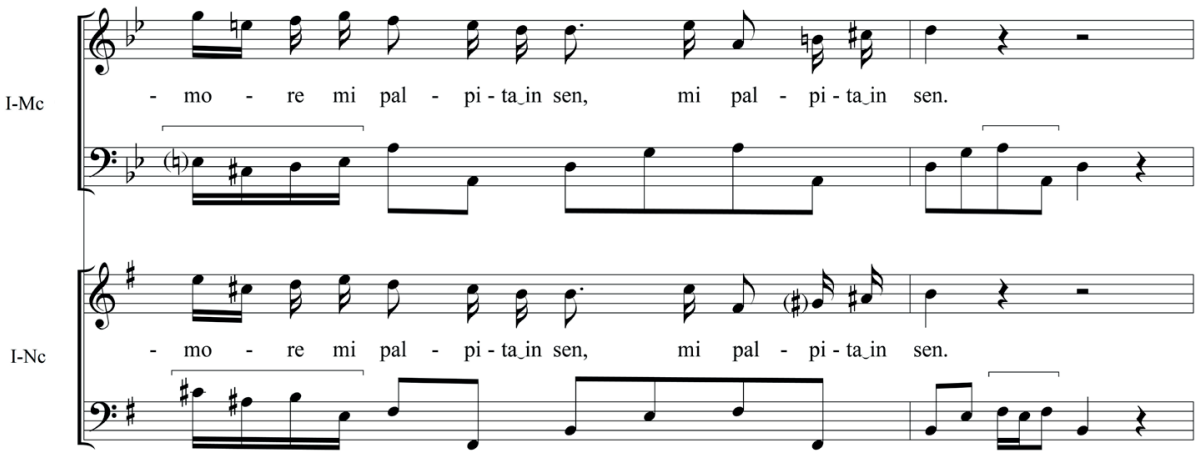

\section{“Del Signor Pistocchino"? Problemi di autenticità}

Un'attenta analisi del contenuto musicale delle due cantate conservate nel fondo Noseda della Biblioteca del Conservatorio di musica "Giuseppe Verdi” di Milano rivela la presenza di alcune debolezze tecniche che mettono fortemente in dubbio la paternità di Pistocchi, soprattutto se confrontate con certe caratteristiche compositive da lui adottate, le cui qualità - riconosciute fin dai suoi contemporanei ${ }^{33}$ - riguardano soprattutto la condotta imitativa delle parti, l'uso del contrappunto rigoroso e la squisita sensibilità di 'disegnare' con la musica il significato dei testi poetici.

Vediamo qualche esempio. Nell'ultima misura del movimento iniziale di Come Dorinda, come la linea del canto registra una successione ravvicinata di due ampi salti melodici, un'ottava discendente (in I-Mc addirittura con ritmo semicroma e croma col punto) seguita da una quinta ascendente, mai riscontrati in una formula cadenzale conclusiva di un recitativo di Pistocchi.

${ }^{33}$ Si veda, per esempio, il lusinghiero commento che Giuseppe Torelli annotò nella lettera del 27 marzo 1700, inviata da Vienna a Giacomo Antonio Perti (maestro di cappella nella Basilica di San Petronio a Bologna): "Circa la stima del caro Pistochino qui in Vienna gli giuro che è grandis[si] ma $\mathrm{p}$ [er]ché toltone la virtù grande del cantare, lui è stimato assais[si]mo nelle sue compositioni, che veram[en]te riusciscono e studiose e vaghe estraordinariam[en]te e non vedo l'ora che lei le veda, e le senta, che so che ancora lei concor[d]erà dispassionatam[en]te con l'opinione e mia e di tutti, che intendono la musica" (I-Bc, P.143.054.a). 


\section{Esempio musicale 4}

Come Dorinda, come, recitativo "Come Dorinda, come", batt. 4-5

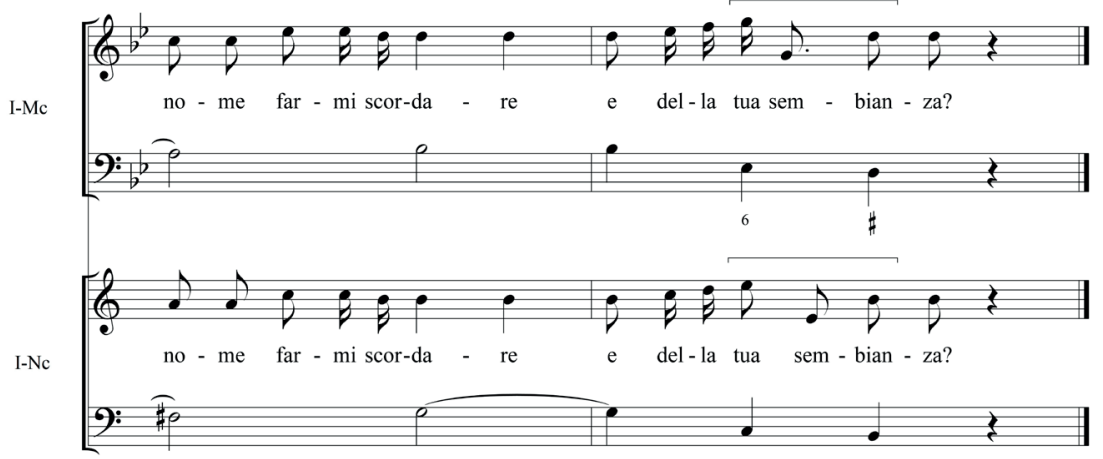

Nelle misure iniziali dell'aria seguente compare una lunga sequenza di tredicesime parallele (su ben undici note) tra la parte vocale e la linea del basso (cfr. incipit ed Esempio musicale 2), una tecnica compositiva mai attestata nella produzione di Pistocchi.

A batt. 4 dell'aria conclusiva, nella parte del basso, si incontra un salto melodico ascendente di settima maggiore, oltretutto in corrispondenza di un brutto parallelismo con la linea vocale, decisamente impossibile da trovare nella produzione di Pistocchi.

\section{Esempio musicale 5}

Come Dorinda, come, aria "In ogni momento", batt. 3-4

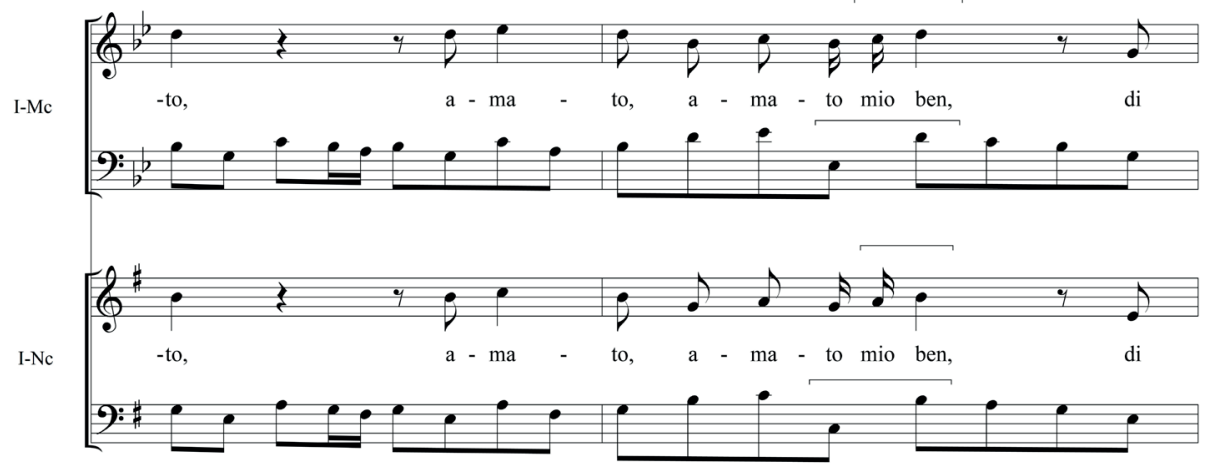

Un'ulteriore caratteristica musicale che accomuna la scrittura delle arie nelle due composizioni del fondo Noseda è la scarsa - o non sempre efficace - interazione contrappuntistica tra la linea del canto e la parte del basso, soprattutto nella cantata Come Dorinda, come, ma anche in E mi lasciasti, ingrata, le cui imitazioni presenti risultano piuttosto deboli soprattutto se confrontate con quelle attestate nelle arie degli Scherzi musicali.

Inoltre, in queste due cantate sono quasi completamente ignorate le introduzioni strumentali nelle arie (ad eccezione di quella poco pregnante in "Un'altra volta" della cantata E mi lasciasti, ingrata), che pure rappresentano normalmente una peculiarità della produzione di Pistocchi, soprattutto nelle cantate manoscritte. 
Un'altra assenza significativa che si riscontra in queste due cantate è costituita dagli 'ariosi' - qui completamente ignorati -, che invece Pistocchi utilizza generalmente alla fine di quasi tutti i suoi recitativi in corrispondenza dell'ultimo verso endecasillabo. ${ }^{34}$ Singolare anche la presenza di una sequenza continua di armonie 'sincopate' in tutte le battute del secondo recitativo di Come Dorinda, come, che non trova riscontro nella produzione di Pistocchi.

E ancora. Varie parole del testo poetico vengono rese in musica in maniera non sempre felice: si vedano, per esempio, in Come Dorinda, come la brutta pronuncia del testo "mia, | ah" (vv. 1-2) a batt. 9 della prima aria, o la sillabazione continua su valori di crome e semicroma nella seconda aria, come pure in E mi lasciasti, ingrata la goffa resa musicale della parola "fuggi" (v. 2) a batt. 2 della prima aria. Inoltre, in entrambe le cantate si nota una resa musicale scarsamente connotativa degli aspetti semantici dei testi poetici: per esempio, parole o locuzioni come "morir" (A1, v. 2), "mi palpita in sen" (A2, v. 7) nella prima cantata, o "mi struggi”" (A1, v. 5), "tormento" (A2, v. 7) nella seconda cantata, non vengono minimamente evidenziate nell'intonazione musicale, contrariamente a quanto fa normalmente Pistocchi nelle sue composizioni in corrispondenza di concetti particolarmente emotivi.

Un altro e importante aspetto strutturale che mette in dubbio la paternità del "Signor Pistocchino" per queste due cantate riguarda l'articolazione tonale dei quattro movimenti di entrambe le composizioni, che è la seguente: ${ }^{35}$

Come Dorinda, come

$\mathrm{R} 1: \mathrm{i} \rightarrow \mathrm{V}$

A1: i

R2: III

A2: $\mathrm{i}$

E mi lasciasti, ingrata

$\mathrm{R} 1: \mathrm{I} \rightarrow \mathrm{VI}$

A1: ii

$\mathrm{R} 2: \mathrm{V} \sharp^{6} \rightarrow \mathrm{I}$

A2: I

Due questioni chiamano l'attenzione: da un lato, l'utilizzo della stessa tonalità per le due arie di Come Dorinda, come, dall'altro, l'impiego del secondo grado minore nella prima aria di E mi lasciasti, ingrata, una cantata in tonalità maggiore.

Riguardo al primo punto, Pistocchi generalmente differenzia le tonalità delle due arie nelle sue cantate articolate in quattro movimenti (RARA o ARAR): in Dolorosa

${ }^{34} \mathrm{Si}$ veda, per esempio, la cantata Pallidetta viola, una composizione che potrebbe risalire agli anni del soggiorno dell'autore a Roma (1693-1694), che contiene ben due ampi 'ariosi' alla fine del secondo e del terzo recitativo (batt. 11-40 e batt. 3-36, rispettivamente).

${ }^{35}$ I gradi tonali maggiori si indicano con numeri romani maiuscoli, quelli minori con numeri romani minuscoli. Nei recitativi la freccia $(\rightarrow)$ indica il percorso tonale: tonalità iniziale e conclusiva. 
partenza, oh Dio, che l'alma, [op. 2] n. 4, in la minore (RARA), la prima aria è al quinto grado minore; in Su la riva del mar tutto dolente, [op. 2] n. 6, in do maggiore (RARA), la prima aria è al terzo grado minore. Anche tra la produzione manoscritta si riscontra una situazione analoga: in Oh, sempre al tuo pastore, in la minore (RARA), la prima aria è al quinto grado minore; in Cinta dal velo usato, in do maggiore ${ }^{36} \mathrm{o}$ in fa maggiore ${ }^{37}$ (RARA), la prima aria è alla dominante; in Bel sospiro che dal seno, in mi minore (ARAR), la seconda aria è al quinto grado minore; in Pria mancare, o bella Clori, in la maggiore (ARAR), la seconda aria è alla sottodominante.

Inoltre, nelle cantate di Pistocchi in tonalità maggiore, escludendo quelle nella struttura ARA (le cui arie - naturalmente - sono entrambe nel tono d'impianto), la varietà delle scelte tonali si attesta esclusivamente su tre possibilità: dominante, sottodominante e terzo grado minore.

La dominante è impiegata nella seconda aria di In su la piaggia aprica, [op. 2] n. 1, in sol maggiore (RARAR), nella prima aria di Oh, delizia degl'orti, oh, amor di Flora, in re maggiore (RARAR), nella seconda aria di Per te forse, mio ben, in re maggiore ${ }^{38} \mathrm{o}$ in sol maggiore ${ }^{39}$ (ARARA), oltreché nella già menzionata prima aria di Cinta dal velo usato.

La sottodominante è adottata nella seconda aria di Fuggi, Fileno, fuggi, in fa maggiore (RARAR), oltreché in Pria mancare, o bella Clori.

Infine, il terzo grado minore si riscontra nella seconda aria di Sovra fiorite piume, in fa maggiore (RARAR), oltreché in Su la riva del mar tutto dolente, [op. 2] n. 6.

\section{Conclusioni}

Le successioni tonali presenti nella cantata Come Dorinda, come (con entrambe le arie nel tono d'impianto) e nella cantata E mi lasciasti, ingrata (in la maggiore, con la prima aria al secondo grado minore) non trovano riscontro nella produzione autentica del "Pistocchino". Se a ciò si aggiunge la presenza di certe peculiarità (o addirittura errori) nell'armonia e nel contrappunto, come pure l'assenza - quasi totale - delle introduzioni strumentali nelle arie e - totale - degli 'ariosi' alla fine dei recitativi, oltre a una scarsa sensibilità di rendere adeguatamente in musica il significato dei testi poetici, risulta evidente che le due cantate per soprano e basso continuo conservate nel fondo Noseda della Biblioteca del Conservatorio di musica "Giuseppe Verdi" di Milano non sono certo composizioni "studiose e vaghe estraordinariamente" tali da poter essere attribuite a Francesco Antonio Pistocchi. ${ }^{40}$

${ }^{36}$ In F-Pn, Rés Vmc Ms 81-9, cc. 21v-24v (RISM ID no. 840027701).

${ }^{37}$ In GB-Lbl, Add. 31594, cc. 17v-20v (RISM ID no. 806046095).

${ }^{38}$ In I-Pca, D.I.1367, pp. 161-168 (RISM deest).

${ }^{39}$ In GB-Lbl, Add. 62102, cc. 48v-50v (numeri invertiti), pp. 29-33 (RISM ID no. 806251381); e in D-SHs, Mus B1:1, cc. 47r-51r, pp. 90-99 (RISM ID no. 250004726).

${ }^{40}$ Naturalmente, resta aperta la questione se la cantata Come Dorinda, come possa essere ascritta o meno a Severo de Luca. 


\section{Bibliografia}

\section{EDIZIONI MUSICALI}

Pistocchi, Francesco Antonio. Chamber Cantata "Pallidetta viola" for Alto voice and basso continuo. A cura di Fabrizio Ammetto e Alejandra Béjar Bartolo. Launton: Edition $\mathrm{HH}$, in preparazione.

- Frammento di una Cantata. Trascrizione di Adolfo Crescentini. Bologna: Achille Tedeschi, [c. 1894].

- Scherzi musicali, [op. II] - Duetti e terzetti, op. III. A cura di Alejandra Béjar Bartolo. Lucca: Libreria Musicale Italiana, 2015.

\section{REGISTRAZIONE DISCOGRAFICA}

Pistocchi, Francesco Antonio. Kantaten und Duette. Bamberg: Cavalli Records, 2001. CCD 312. http://opac2.icbsa.it/vufind/Record/IT-DDS0000046020000000.

\section{LETTERATURA}

Béjar Bartolo, Alejandra. "Una cantata, dos voces (¿soprano o alto?), cuatro (¿o cinco?) fuentes: Pallidetta viola de Francesco Antonio Pistocchi”. In Aspectos de la investigación musicológica actual: compositores, formas musicales, ejecución, improvisación, arte y paisaje sonoro, a cura di Fabrizio Ammetto, 19-32. Guanajuato: Universidad de Guanajuato, 2019.

- e Fabrizio Ammetto. “Aspectos de análisis textual y musical en los 'Scherzi musicali’ (1698) de Francesco Antonio Pistocchi”. Per Musi 18, n. 37 (2017): 1-18.

—. "Las composiciones de Francesco Antonio Pistocchi (1659-1726): listado actualizado de su obra". Revista Iberoamericana de las Ciencias Sociales y Humanísticas 6, n. 11 (2017): 313-335. https://doi.org/10.23913/ricsh.v6i11.120.

Hanley, Edwin H. "Alessandro Scarlatti's cantate da camera: A Bibliographical Study". Diss. dottorale, Yale University, 1963.

Mischiati, Oscar. Indici, cataloghi e avvisi degli editori e librai musicali italiani dal 1591 al 1798. Firenze: Leo S. Olschki, 1984.

Rostirolla, Giancarlo, ed. Alessandro Scarlatti: catalogo generale delle opere. Torino: ERI, 1972.

Silvani, Giuseppe Antonio. Indice dell'opere di musica sin'hora stampate in Bologna. Bologna: Giuseppe Antonio Silvani, 1724.

Talbot, Michael, ed. Aspects of the Secular Cantata in Late Baroque Italy. Farnham: Ashgate, 2009.

Talbot, Michael. The Chamber Cantatas of Antonio Vivaldi. Woodbridge: Boydell Press, 2006. 


\section{KANTATI V MILANU, NAPAČNO PRIPISANI \\ FRANCESCU ANTONIU PISTOCCHIJU}

Povzetek

V Nosedini zbirki knjižnice glasbenega konservatorija »Giuseppe Verdi« v Milanu sta v rokopisu ohranjeni dve kantati za sopran in basso continuo, pripisani Francescu Antoniu Pistocchiju, z naslovoma Come Dorinda, come in E mi lasciasti, ingrata. Obe sta zgrajeni v zaporedju recitativ-arija-recitativ-arija (RARA). Prva skladba se je ohranila v še enem rokopisu v knjižnici neapeljskega glasbenega konservatorija San Pietro a Majella (toda v drugi tonaliteti), njeno avtorstvo pa je pripisano Severu de Luci. Natančna analiza obeh kantat je razkrila različne kompozicijskotehnične slabosti (v nekaterih primerih celo prave napake), ki izključujejo Pistocchijevo avtorstvo, saj gre za skladatelja, čigar glasbeno mojstrstvo je splošno priznano že vse od njegovega časa. Med najbolj problematičnimi vidiki teh dveh del so slaba kontrapunktična interakcija med vokalnim delom in basovsko linijo, popolna odsotnost ariosov v recitativih in skoraj popolna odsotnost instrumentalnih uvodov v arije (ki so v izvirnih Pistocchijevih kantatah vedno prisotni). Poleg tega se težave včasih pojavijo zaradi slabe uglasbitve pesemskega besedila in splošno nezadovoljivega glasbenega podajanja semantične plasti pesmi. Najpomembneje pa je, da kantati v svojih štirih stavkih izkazujeta kombinacijo tonalitet, ki je v podobnih Pistocchijevih delih ne najdemo: v kantati Come Dorinda, come je v obeh arijah uporabljena ista tonaliteta, medtem ko je v prvi ariji kantate E mi lasciasti, ingrata (napisane v duru) uporabljena (molova) subdominantna paralela. V svojih kantatah v duru je namreč v arijah Pistocchi poleg tonike uporabljal samo dominanto, subdominanto in dominantno paralelo. 